International Journal of Pure and Applied Mathematics

Volume 98 No. 1 2015, 145-156

ISSN: 1311-8080 (printed version); ISSN: 1314-3395 (on-line version)

url: http://www.ijpam.eu

doi: http://dx.doi.org/10.12732/ijpam.v98i1.12

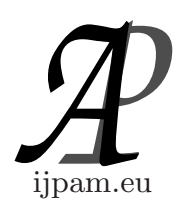

\title{
SUFFICIENT CONDITIONS FOR OSCILLATION OF SECOND ORDER NEUTRAL ADVANCED DIFFERENCE EQUATIONS
}

\author{
A. Murugesan ${ }^{1 \S}, \mathrm{K}$. Ammamuthu ${ }^{2}$ \\ ${ }^{1}$ Department of Mathematics \\ Government Arts College (Autonomous) \\ Salem - 636 007, Tamil Nadu, INDIA \\ ${ }^{2}$ Department of Mathematics \\ Arignar Anna Government Arts College \\ Vadachennaimalai, Attur-636 121 \\ Tamil Nadu, INDIA
}

\begin{abstract}
The aim of this paper is to study the oscillation of the second order neutral advanced difference equations

$$
\Delta(r(n) \Delta[x(n)+p(n) x(\tau(n))])+q(n) x(\sigma(n))=0, \quad n=0,1,2, \ldots
$$

Obtained results are based on the new comparison theorems that enable us to reduce problem of the oscillation of the second order equation the oscillation of the first order equations. Obtained comparison principles essentially simplify the examination of the studied equations.
\end{abstract}

AMS Subject Classification: 39A10, 39A12

Key Words: second order difference equations, neutral advanced difference equation, oscillation and nonoscillation properties

\section{Introduction}

In this paper, we are concerned with the oscillation of the solutions of second

Received: September 20, 2014

(c) 2015 Academic Publications, Ltd.

${ }^{\S}$ Correspondence author url: www.acadpubl.eu 
order neutral advanced equations of the form

$$
\Delta(r(n) \Delta[x(n)+p(n) x(\tau(n))])+q(n) x(\sigma(n))=0, \quad n=0,1,2, \ldots,
$$

where $\Delta$ is the forward difference operator given by $\Delta x(n)=x(n+1)-x(n)$, $\{p(n)\}_{n>0}, \quad\{q(n)\}_{n>0}$ and $\quad\{r(n)\}_{n>0}$ assumed to be infinite sequences of real numbers with $q(n)>0, r(n)>0$. Also $\{\tau(n)\}_{n>0}$ and $\{\sigma(n)\}_{n \geq 0}$ are sequences of positive integers. Further the following conditions are assumed for its use in the sequel.

$\left(\mathrm{H}_{1}\right) \sigma(n)>n+1$ and $\{\sigma(n)\}_{n \geq 0}$ is nondecreasing;

$\left(\mathrm{H}_{2}\right) \lim _{n \rightarrow \infty} \tau(n)=\infty$;

$\left(\mathrm{H}_{3}\right) \Delta \tau(n) \geq \tau_{0}>0$

$\left(\mathrm{H}_{4}\right) \tau \circ \sigma=\sigma \circ \tau$;

$\left(\mathrm{H}_{5}\right) R(n)=\sum_{s=0}^{n} \frac{1}{r(s)} \rightarrow \infty$ as $n \rightarrow \infty$.

Let $\{x(n)\}$ be a real sequence. We will also define a companion or associated sequence $\{z(n)\}$ of it by

$$
z(n)=x(n)+p(n) x(\tau(n)), \quad n \geq 0,
$$

where $\{p(n)\}$ and $\{\tau(n)\}$ have been defined above.

Let $n_{0}$ be a fixed nonnegative integer. By a solution of (1), we mean a nontrivial real sequence $\{x(n)\}$ which is defined for $n \geq \min \left\{n_{0}, \tau\left(n_{0}\right)\right\}$ and satisfies the equation (1) for $n \geq n_{0}$. A solution $\{x(n)\}$ of (1) is said to be oscillatory if for every positive integer $N>0$, there exists an $n \geq N$ such that $x(n) x(n+1) \leq 0$, otherwise $\{x(n)\}$ is said to be nonoscillatory. Equation (1) is said to be oscillatory if all its solutions are oscillatory.

Recently, there has been a lot of interest in studying the oscillatory behavior of difference equations. See, for example [1-6, 10] and references cited there in. They have mainly concerned with the oscillation and nonoscillatory of solutions of (1). The advanced equations have wide use. For example, the population of the future limit to population growth can be described through (1) with $p(n) \equiv 0$. Sternal et al. [9] showed that $-1 \leq p_{1} \leq p_{n} \leq 0$ together with $\sum_{n=0}^{\infty} \frac{1}{r_{n}}=\infty$ and $\sum_{n=0}^{\infty} q_{n}=\infty$ guarantee the oscillation of unbounded solutions of the neutral equation

$$
\Delta\left(r_{n} \Delta\left[u_{n}+p_{n} u_{n-k}\right]\right)+q_{n} f\left(u_{n-l}\right)=0 .
$$


For the same equation R.N. Rath et al. [8] established oscillation criteria . This results has been improved and generalized by other authors. We mention Tripathy [11] who studied oscillation of

$$
\Delta\left(r_{n} \Delta\left[y_{n}+p_{n} y_{n-m}\right]\right)+f_{n} H_{1}\left(y_{n-k_{1}}\right)-g_{n} H_{2}\left(y_{n-k_{2}}\right)=q_{n}
$$

under the conditions

$$
\sum_{n=0}^{\infty} \frac{1}{r_{n}}<\infty \quad \text { and } \quad \sum_{n=0}^{\infty} \frac{1}{r_{n}}=\infty .
$$

Zhang et al. [12] established oscillation criteria for the equation (1) with

$$
p(n) \equiv 0 \quad \text { and } \quad \sum_{n=n_{0}}^{\infty} q(n)=\infty .
$$

In this paper, we discuss the oscillation of solutions of (1). We obtain some better sufficient conditions for (1) to be oscillatory. They are delicate criteria. Our technique permits to relax restrictions usually imposed on the coefficients of (1). So that our results are of high generality and can be easily extended also to the nonlinear neutral difference equations. Obtained results are easily applicable and are illustrated on a suitable example.

In the sequel for convenience when we write a functional inequality without specifying its domain of validity, we assume that it holds for all sufficiently large positive integer $n$.

\section{Some Lemmas}

First we state a lemma which is due to [5].

Lemma 1. Assume that $\tau(n)=n-\tau, \tau$ is a positive integer and $-1<p \leq$ $p(n) \leq 0$. Assume further that $\{x(n)\}$ is an eventually positive solution of (1) and $\{z(n)\}$ is its associated sequence defined by (2). If $\{\Delta z(n)\}$ is eventually negative or if $\lim _{n \rightarrow \infty} \sup x(n)>0$, then $\{z(n)\}$ is eventually positive.

Lemma 2. If $\{x(n)\}$ is an eventually positive solution of (1), then its associated sequence $\{z(n)\}$ defined by (2) satisfies $z(n)>0, r(n) \Delta z(n)>0$ and $\Delta(r(n) \Delta z(n))<0$ eventually. 
Proof. Assume that $\{x(n)\}$ is an eventually positive solution of (1). Then it follows from (1) that

$$
\Delta(r(n) \Delta z(n))=-q(n) x(\sigma(n))<0
$$

Consequently, $\{r(n) \Delta z(n)\}$ is decreasing and thus either $\Delta z(n)>0$ or $\Delta z(n)<$ 0 , eventually. If we let $\Delta z(n)<0$, then also $r(n) \Delta z(n)<-c<0$ and summing this from $n_{1}$ to $n-1$, we have

$$
z(n) \leq z\left(n_{1}\right)-c \sum_{s=n_{1}}^{n-1} \frac{1}{r(s)} \rightarrow-\infty \quad \text { as } \quad n \rightarrow \infty
$$

This contradicts the positivity of $\{z(n)\}$ and the proof is complete.

Lemma 3. If $\tau(n)=n-\tau, \tau$ is a positive integer, $-1 \leq p \leq p(n) \leq$ 0 and $\{x(n)\}$ is an eventually positive solution of (1) such that $\lim _{\sup _{n \rightarrow \infty}}$ $x(n)>0$, then its associated sequence $\{z(n)\}$ defined by (2) satisfies $z(n)>0$, $r(n) \Delta z(n)>0$ and $\Delta(r(n) \Delta z(n))<0$, eventually.

Proof. Assume that $\{x(n)\}$ is an eventually positive solution of (1) such that

$$
\limsup _{n \rightarrow \infty} x(n)>0
$$

Then it follows from (1) that

$$
\Delta(r(n) \Delta z(n))=-q(n) x(\sigma(n))<0 .
$$

Consequently $\{r(n) \Delta z(n)\}$ is decreasing and thus either $\Delta z(n)>0$ or $\Delta z(n)<$ 0 , eventually. If we let $\Delta z(n)<0$, then by Lemma $1, z(n)>0$ eventually. Then also $r(n) \Delta z(n)<-c<0$ and summing this from $n_{1}$ to $n-1$, we have

$$
z(n) \leq z\left(n_{1}\right)-c \sum_{s=n_{1}}^{n-1} \frac{1}{r(s)} \rightarrow-\infty \quad \text { as } \quad n \rightarrow \infty
$$

This contradicts the positivity of $\{z(n)\}$ and hence $\Delta z(n)>0$. Since limsup $\operatorname{su}_{n \rightarrow \infty}$ $x(n)>0$, by Lemma 1 we have $z(n)>0$ eventually and the proof is complete.

A slight modification in the proof of the Theorem 3 in [7] leads to the following lemma about the advanced difference inequality. 
Lemma 4. Consider the advanced difference inequality

$$
\Delta x(n)-q(n) x(\sigma(n)) \geq 0 .
$$

where $\{q(n)\}$ and $\{\sigma(n)\}$ are defined in (1). If

$$
\liminf _{n \rightarrow \infty} \frac{1}{\sigma(n)-n-1} \sum_{s=n+1}^{\sigma(n)-1} q(s)>\limsup _{n \rightarrow \infty} \frac{(\sigma(n)-n-1)^{\sigma(n)-n-1}}{(\sigma(n)-n)^{\sigma(n)-n}},
$$

then (3) has no eventually positive solution.

For our further references, let us denote

$$
\begin{gathered}
Q(n)=\min \{q(n), q(\tau(n))\} \\
Q_{1}(n)=\frac{1}{r(n)} \sum_{s=n}^{\infty} Q(s) ;
\end{gathered}
$$

and

$$
Q_{2}(n)=Q(n)\left(R(n)-R\left(n_{1}-1\right)\right),
$$

where $n \geq n_{1}, n_{1}$ is a large enough.

\section{Main Results}

Theorem 5. Let $\tau(n) \geq n$ and $0 \leq p(n) \leq p_{0}<\infty$. Assume that at least one of the first order advanced difference inequalities

$$
\begin{aligned}
& \Delta w(n)-\frac{\tau_{0}}{\tau_{0}+p_{0}} Q_{1}(n) w(\sigma(n)) \geq 0 ; \\
& \Delta w(n)-\frac{\tau_{0}}{\tau_{0}+p_{0}} Q_{2}(n) w(\sigma(n)) \geq 0 ;
\end{aligned}
$$

has no positive solution. Then (1) is oscillatory.

Proof. Assume that $\{x(n)\}$ is an eventually positive solution of (1). Then from (2), we have

$$
z(\sigma(n))=x(\sigma(n))+p(\sigma(n)) x(\tau(\sigma(n))) \leq x(\sigma(n))+p_{0} x(\sigma(\tau(n)))
$$

where we used to the hypothesis $\left(H_{4}\right)$.

On the other hand, it follows from (1) that 


$$
\Delta(r(n) \Delta z(n))+q(n) x(\sigma(n))=0 ;
$$

and more over, taking $\left(\mathrm{H}_{3}\right)$ into account, we have

$$
\begin{aligned}
0 & =\frac{1}{\Delta z(n)} \Delta\left(r(\tau(n)) \frac{\Delta z(\tau(n))}{\Delta z(n)}\right)+q(\tau(n)) x(\sigma(\tau(n))) \\
& \geq \frac{p_{0}}{\tau_{0}} \Delta\left(r(\tau(n)) \frac{\Delta z(\tau(n))}{\Delta z(n)}\right)+p_{0} q(\tau(n)) x(\sigma(\tau(n))) .
\end{aligned}
$$

Combining (10) and (11), we are lead to

$$
\begin{aligned}
\Delta(r(n) \Delta z(n))+\frac{p_{0}}{\tau_{0}} \Delta & \left(r(\tau(n)) \frac{\Delta z(\tau(n))}{\Delta \tau(n)}\right) \\
& +q(n) x(\sigma(n))+p_{0} q(\tau(n)) x(\sigma(\tau(n))) \leq 0
\end{aligned}
$$

which in view of (9) and (4) provides

$$
\Delta(r(n) \Delta z(n))+\frac{p_{0}}{\tau_{0}} \Delta\left(r(\tau(n)) \frac{\Delta z(\tau(n))}{\Delta \tau(n)}\right)+Q(n) z(\sigma(n)) \leq 0 .
$$

Summing the previous inequality from $n$ to $\infty$, we get

$$
r(n) \Delta z(n)+\frac{p_{0}}{\tau_{0}}\left(r(\tau(n)) \frac{\Delta z(\tau(n))}{\Delta \tau(n)}\right) \geq \sum_{s=n}^{\infty} Q(s) z(\sigma(s)) .
$$

On the other hand, since $\{r(n) \Delta z(n)\}$ is decreasing and $\tau(n) \geq n$, it follows from (13) that

$$
r(n) \Delta z(n)\left(1+\frac{p_{0}}{\tau_{0}}\right) \geq \sum_{s=n}^{\infty} Q(s) z(\sigma(s)) .
$$

Using that $\{z(\sigma(n))\}$ is increasing, an summation from $n_{1}$ to $n-1$, yields,

$$
\begin{aligned}
z(n) & \geq \frac{\tau_{0}}{\tau_{0}+p_{0}} \sum_{k=n_{1}}^{n-1} \frac{1}{r(k)} \sum_{s=k}^{\infty} Q(s) z(\sigma(s)) \\
& \geq \frac{\tau_{0}}{\tau_{0}+p_{0}} \sum_{k=n_{1}}^{n-1} \frac{z(\sigma(k))}{r(k)} \sum_{s=k}^{\infty} Q(s) .
\end{aligned}
$$

That is,

$$
z(n) \geq \frac{\tau_{0}}{\tau_{0}+p_{0}} \sum_{k=n_{1}}^{n-1} Q_{1}(k) z(\sigma(k))
$$


Let us denote the right hand side of (15) by $w(n)$. Then $w(n)>0$ and using $z(n) \geq w(n)$, one can see that

$$
\Delta w(n)=\frac{\tau_{0}}{\tau_{0}+p_{0}} Q_{1}(n) z(\sigma(n)) \geq \frac{\tau_{0}}{\tau_{0}+p_{0}} Q_{1}(n) w(\sigma(n)) .
$$

Thus $\{w(n)\}$ is a positive solution of (7). This contradicts our assumptions and thus the absence of the eventually positive solutions of (7) implies the oscillatory of (1).

Now, we shall show that the absence of the eventually positive solutions of (8) also yields the oscillation of (1). An summation of (14) from $n_{1}$ to $n-1$, provides

$$
\begin{gathered}
z(n) \geq \frac{\tau_{0}}{\tau_{0}+p_{0}} \sum_{k=n_{1}}^{n-1} \frac{1}{r(k)} \sum_{s=k}^{\infty} Q(s) z(\sigma(s)) \\
\geq \frac{\tau_{0}}{\tau_{0}+p_{0}} \sum_{k=n_{1}}^{n-1} \frac{1}{r(k)} \sum_{s=k}^{\infty} Q(s) z(\sigma(s)) \\
=\frac{\tau_{0}}{\tau_{0}+p_{0}} \sum_{s=n_{1}}^{n-1} Q(s) z(\sigma(s)) \sum_{k=n_{1}}^{\infty} \frac{1}{r(k)} .
\end{gathered}
$$

That is,

$$
z(n) \geq \frac{\tau_{0}}{\tau_{0}+p_{0}} \sum_{s=n_{1}}^{n-1} Q_{2}(s) z(\sigma(s)) .
$$

Let us denote the right hand side of $(16)$ by $w(n)$. Then $w(n)>0$ and using that $z(n) \geq w(n)$; one can see that $\{w(n)\}$ is an eventually positive solution of (8). This is a contradiction and the proof is complete now.

Theorem 6. Let $\tau(n) \geq n$ and $0 \leq p(n) \leq p_{0}<\infty$. Assume that at least one of the following conditions

$$
\begin{array}{r}
\liminf _{n \rightarrow \infty} \frac{1}{\sigma(n)-n-1} \sum_{k=n+1}^{\sigma(n)-1} Q_{1}(k)>\left(\frac{\tau_{0}+p_{0}}{\tau_{0}}\right) \\
\limsup _{n \rightarrow \infty} \frac{(\sigma(n)-n-1)^{\sigma(n)-n-1}}{(\sigma(n)-n)^{\sigma(n)-n}} ;
\end{array}
$$




$$
\begin{array}{r}
\liminf _{n \rightarrow \infty} \frac{1}{\sigma(n)-n-1} \sum_{k=n+1}^{\sigma(n)-1} Q_{2}(k)>\left(\frac{\tau_{0}+p_{0}}{\tau_{0}}\right) \\
\limsup _{n \rightarrow \infty} \frac{(\sigma(n)-n-1)^{\sigma(n)-n-1}}{(\sigma(n)-n)^{\sigma(n)-n}}
\end{array}
$$

holds. Then (1) is oscillatory.

Proof. Lemma 4 guarantees that (7) and (8) have no eventually positive solutions provided that (17) and (18) hold respectively. The assertion now follows from Theorem 5 .

For our incoming references, let us denote

$$
Q_{3}(n)=\frac{\Delta \tau(n)}{r(\tau(n))} \sum_{s=n}^{\infty} Q(s) ; \quad Q_{4}(n)=Q(n)\left(R(\tau(n))-R\left(\tau\left(n_{1}-1\right)\right)\right) .
$$

Theorem 7. Assume that $\tau(n)=n-\tau$ and $\sigma(n)=n+\sigma$ where $\tau$ and $\sigma$ are positive integers and $0 \leq p(n) \leq p_{0}<\infty$. Assume further that at least one of the first order advanced difference inequalities

$$
\begin{aligned}
& \Delta w(n)-\frac{1}{1+p_{0}} Q_{3}(n) w(n+\tau+\sigma) \geq 0 \\
& \Delta w(n)-\frac{1}{1+p_{0}} Q_{4}(n) w(n+\tau+\sigma) \geq 0
\end{aligned}
$$

has no eventually positive solution. Then (1) is oscillatory.

Proof. Assume that (20) has no eventually positive solution. Without loss of generality, we suppose that $\{x(n)\}$ is an eventually positive solution of (1). Then its associated sequence $\{z(n)\}$ defined by (2) satisfies (13). That is,

$$
r(n) \Delta z(n)+p_{0} r(n-\tau) \Delta z(n-\tau) \geq \sum_{s=n}^{\infty} Q(s) z(s+\sigma),
$$

where $\tau_{0}=1$ and $\Delta \tau(n)=1$.

On the other hand, since $\{r(n) \Delta z(n)\}$ is decreasing, then it follows from (22) that

$$
r(n-z) \Delta z(n-\tau)\left(1+p_{0}\right) \geq \sum_{s=n}^{\infty} Q(s) z(s+\sigma)
$$


Multiplying by $\frac{1}{r(n-\tau)}$ and then summing from $n_{1}$ to $n-1$, we get

$$
\begin{aligned}
z(n-\tau) \geq \frac{1}{1+p_{0}} \sum_{k=n_{1}}^{n-1} \frac{1}{r(k-z)} \sum_{s=k}^{\infty} Q(s) z(s+\sigma) \\
\quad \geq \frac{1}{1+p_{0}} \sum_{k=n_{1}}^{n-1} z(k+\sigma) \frac{1}{r(k-z)} \sum_{s=k}^{\infty} Q(s) .
\end{aligned}
$$

That is,

$$
z(n-\tau) \geq \frac{1}{1+p_{0}} \sum_{k=n_{1}}^{n-1} Q_{3}(k) z(k+\sigma) .
$$

Let us denote the right hand side of $(24)$ by $w(n)$. Noting that $z(n-\tau) \geq$ $w(n)$. One can see that $\{w(n)\}$ is an eventually positive solution of (20). This contradicts our assumptions and thus the absence of the positive solutions of (20) implies the oscillation of (1).

Now we shall show that the absence of the positive solutions of (21) implies the oscillation of (1). An summation of (23) from $n_{1}$ to $n-1$, gives

$$
\begin{aligned}
z(n-\tau) & \geq \frac{1}{1+p_{0}} \sum_{k=n_{1}}^{n-1} \frac{1}{r(k-\tau)} \sum_{s=k}^{\infty} Q(s) z(s+\sigma) \\
& \geq \frac{1}{1+p_{0}} \sum_{k=n_{1}}^{n-1} \frac{1}{r(k-\tau)} \sum_{s=k}^{n-1} Q(s) z(s+\sigma) \\
& =\frac{1}{1+p_{0}} \sum_{s=n_{1}}^{n-1} Q(s) z(s+\sigma) \sum_{k=n_{1}-\tau}^{s-\tau} \frac{1}{r(k)}
\end{aligned}
$$

That is,

$$
z(n-\tau) \geq \frac{1}{1+p_{0}} \sum_{s=n_{1}}^{n-1} Q_{4}(s) z(s+\sigma) .
$$

Let us denote the right hand side of (25) by $w(n)$. Then $w(n)>0$ and using that $z(n-\tau) \geq w(n)$, one can see that $\{w(n)\}$ is an eventually positive solution of (21). This is a contradiction and the proof is complete now. 
Theorem 8. Assume that $\tau(n)=n-\tau, \sigma(n)=n+\sigma$, where $\tau$ and $\sigma$ are positive integers and $0 \leq p(n) \leq p_{0}<\infty$. Assume further that at least one of the following conditions

$$
\begin{aligned}
& \liminf _{n \rightarrow \infty} \sum_{s=n+1}^{n+\tau+\sigma-1} Q_{3}(s)>\left(1+p_{0}\right)\left(\frac{\tau+\sigma-1}{\tau+\sigma}\right)^{\tau+\sigma} ; \\
& \liminf _{n \rightarrow \infty} \sum_{s=n+1}^{n+\tau+\sigma-1} Q_{4}(s)>\left(1+p_{0}\right)\left(\frac{\tau+\sigma-1}{\tau+\sigma}\right)^{\tau+\sigma} ;
\end{aligned}
$$

holds. Then (1) is oscillatory.

Proof. Lemma 4 guarantees that (20) and (21) have no eventually positive solutions provided that (26) and (27) hold respectively. The assertion now follows from Theorem 7 .

Theorem 9. Assume that $\tau(n)=n-\tau, \sigma(n)=n+\sigma$, where $\tau$ and $\sigma$ are positive integers and $-1<p \leq p(n) \leq 0$. Assume further that at least one of the first order advanced difference inequalities (20) and (21) has no eventually positive solution. Then every solution of (1) is either oscillatory or tends to zero as $n \rightarrow \infty$.

Theorem 10. Assume that $\tau(n)=n-\tau, \sigma(n)=n+\sigma$, where $\tau$ and $\sigma$ are positive integers and $-1<p \leq p(n) \leq 0$. Assume further that at least one of the conditions (26) and (27) holds. Then every solution of (1) is either oscillatory or tends to zero as $n \rightarrow \infty$.

\section{Proofs of Theorem 9 and 10.}

Without loss of generality, we may assume that $\{x(n)\}$ is an eventually positive solution of (1) such that $\lim _{\sup } \rightarrow \infty x(n)>0$. Then, by Lemma 3 , we have $z(n)>0, r(n) \Delta z(n)>0$ and $\Delta(r(n) \Delta z(n))>0$, eventually.

Theorem 9 and Theorem 10 can be proved by applying the procedures that are used in the Theorem 7 and 8 respectively.

Example 11. We consider the second order neutral advanced difference equation

$$
\Delta\left(\frac{1}{2^{n}} \Delta\left[x(n)+\frac{1}{n+1} x(n+1)\right]\right)+\frac{1}{2^{n}} x(n+2)=0 ; \quad n=0,1,2 \ldots
$$


where $p(n)=\frac{1}{n+1}, r(n) \frac{1}{2^{n}}, q(n)=\frac{1}{2^{n}}, \sigma(n)=n+2$ and $\tau(n)=n+1$. Clearly, $p_{0}=1, \tau_{0}=1, Q(n)=\frac{1}{2^{n+1}}$ and $Q_{1}(n)=1$.

Also,

$$
\begin{gathered}
\liminf _{n \rightarrow \infty} \frac{1}{\sigma(n)-n-1} \sum_{s=n+1}^{\sigma(n)-1} Q_{1}(s)=1 \\
>\left(\frac{\tau_{0}+p_{0}}{\tau_{0}}\right) \lim _{n \rightarrow \infty} \sup \frac{(\sigma(n)-n-1)^{\sigma(n)-n-1}}{(\sigma(n)-n)^{\sigma(n)-1}} \\
=\frac{1}{2} .
\end{gathered}
$$

Then by Theorem 6, every solution of (28) is oscillatory.

Remark 12. All our conclusions can be very easily extend to nonlinear neutral difference equations of the form

$$
\Delta(r(n) \Delta[x(n)+p(n) x(\tau(n))]+q(n) f(x(\sigma(n))=0 .
$$

Adding the additional condition

$$
\frac{f(x)}{x} \geq \lambda
$$

the reader can verify that our results here hold also for (29), provided that we replace in the assumption of our achievements the sequences $\{q(n)\}$ by $\{\lambda q(n)\}$.

\section{References}

[1] R.P. Agarwal, M. M. S. Manuel, E. Thandapani, Oscillatory and nonoscillatory behavior of second order neutral delay difference equations, Math. Comput. Modelling, 24(1996) 5-11, doi:10.1016/0895-7177(96)000076-3. .

[2] Bi Ping, Maoan Han, Oscillation of second order difference equations with advanced argument, Proceedings of the fourth international conference on Dynamical Systems and Differential equations, Wilmington, NC, USA, May 24-27(2002) 108-112.

[3] S.S. Cheng, Y.Z. Lin, Complete characterizations of an oscillatory neutral difference equation, J. Math. Anal. Appl., 221(1998) 73-91, doi: 10.1006/jmaa.1997.5341. 
[4] Horng-Jaan Li, Cheh-Chih Yeh, Oscillation criteria for second order neutral delay difference equations, Comput. Math. Appl., 36(1998) 123-132, doi: 10.1016/S0898-1221(98)80015-1.

[5] W. T. Li, S.S. Cheng, Classifications and existence of positive solutions of second order nonlinear neutral difference equations, Funkcial. Ekvac., 40(1997) 371-393.

[6] A. Murugesan, Oscillation of neutral advanced difference equation, Glob. J. Pure. Appl. Math., 9(2013) 83-92.

[7] Ch. G. Philos, On oscillations of some difference equations, Funkcial. Ekvac., 34(1991) 157-172.

[8] R.N. Rath, S. Padhi, B. L. S. Baric, Oscillatory and asymptotic behaviour of a homogenous neutral delay difference equation of second order, Academia Sinica (New series), 3(2008) 453-467.

[9] A. Sternal, B. Szmanda, Asymptotic and oscillatory behaviour of certain difference equations, Le Mathematiche, 51(1996) 77-86.

[10] E. Thandapani, P. Sundaram, J. R. Greaf, P. W. Spikes, Asymptotic behaviour and oscillation of solutions of neutral delay difference equations of arbitrary order, Math. Slovaca, 47(1997) 539-551.

[11] A.K.Tripathy, Oscillatory behavior of a class of nonlinear second order mixed difference equations, Electron. J. Qual. Theory Differ. Equ., 48(2010) 1-19.

[12] Z. Zhang, Q. Li, Oscillation theorems for second order advanced functional difference equations, Comput. Math. Appl., 36(1998) 11-18,doi: 10.1016/S0898-1221(98)00157-6. 